\title{
Autism and Nutritional Biotherapy
}

\author{
Ferrara Lydia ${ }^{1}$, Flammia Aurora ${ }^{2}$ \\ ${ }^{I}$ Department of Pharmacy, University of Naples "Federico II," via Domenico Montesano 49, 80131, Naples, \\ Italy. \\ ${ }^{2}$ ISFOM, Istituto Formazione Musicoterapia, corso Vittorio Emanuele 87, 80122, Naples, Italy
}

\begin{abstract}
Alteration of the perception of the taste of foods is called dysgeusia and can be due to various factors: alteration of the gustative papillae that cover all the language and that they are responsible of the reception of the savours; reduction of some oligoelements that are necessary for the perception of the taste (es. zinc); or lack of some enzymes; reduced salivation; alteration of the cerebral mechanisms that regulate the perception of the taste; alteration of the sense of the smell one that it does not to allow it smells it and to invite to the assay of the food. Some autistic subjects introduce towards it smell an amazing olfactory ability, while others can feel aversion for smell and strong savours and therefore are much selective in the feeding, compromising, sometimes, the necessary nutritional intake with consequent decrease of the weight them and damages for the health. A valid aid, in such cases, comes offered from the nutritional biotherapy that takes cure to introduce the aliments under various aspects rendering them more attractive, stimulating the curiosity of the subject. To propose food as medicine is useful is to the nutritional purposes is to the prevention of the onset of chronic pathologies contributing to the good function of the organs and to the protection of the natural defenses
\end{abstract}

Keywords: autism, sense of taste, intestinal dysbiosis, nutritional biotherapy

\section{Introduction}

The autistic child is a child cerebral palsy, who presentes sensory channels alters (the sight, the hearing, the tact, taste and the smell one) not having the ability, because of serious neurological problems, to transform the feelings in complete perceptions and correctly works them. The perceptions are thereforee ambiguous and determine an anomalous behavior. Sensory channels in autistic subjects can be altered in three different ways: Hyper, entered indiscriminate of information and too many stimulations they reach the brain at the same time; ipo entered deficit of information with deficiency of cerebral stimulation; white noise, distortion of information in entrance because of the superposition of stimulations that reach the brain with stimulations created from the same sensory channels (Delacato C.H. 1996)[1].

In the autistic subjects the sensory alteration of the taste is one of the causes for which an autistic child introduces problems of feeding for which it is necessary to intervene not only therapeutic, but also by calling for a greater interest in the food. The autistic child with problems gustatory not only has undernourished for a defective or inadequate feeding, but he can, for example, eating non-edible substances as detergents or soap, compromising the own health; it can be selective, nourishing itself for long periods always with the same food; even, in some cases can regurgitate the ingested food and then reassume it. The main organ of taste is the tongue, on the whose surface are present approximately10,000 gustative papillae that are responsible of the perception of the four tastes: sour, salty, bitter sweet. The tip of the tongue is sensitive above all to salty and sweet; the side parts to the acid; the posterior part to the bitter flavors. Anything has a taste, if put in the mouth, comes second estimated from the tongue these four tastes; moreover it is extremely sensitive also to the tact, distinguishing the consistency of foods and sometimes turns out difficult to separate the gustative function from that tactile one.

The children hyper taste they have feeding problems because of their limited tolerance to the taste variations; they generally eat a lot little and little tasty food; they mostly use the tip of the tongue in order to taste. The hildren ipo taste, taste all, carry all to the mouth, make to slide the tongue on the objects and also on the persons in order to learn more on they. They stretch to use the posterior part and the sides of the tongue where are present the taste acid and bitter. The children who introduce the anomaly taste white noise have always a taste of bottom in their mouth, like if their gustatory experiences were generated from the inside. They in fact suck the own tongue, especially to sides, with consequent in largery of the same one and are generally little inclined to the food.

The habits and the alimentary preferences of the autistic children are atypical and often considered a problem for the corrected nutrition, because they can provoke to malnutrition and devoid nutritional statuses. The generally manifest choice with a preference for some particular foods, and is based on the consistency, the color and shape of the foods; usually it is turned towards dry foods or semiliquid. Greater part of these children 
has moreover recurrent behaviors in the moment of the meal, like touching fleetingly the food that comes they proposed, with this in mind to try of the consistency, testing it to oral level, to smell it and only later on to eat it. For other children a lot is a routine behavior over a long time span for chewing the food, untilthet it comes swallowed for accidental ingestion rather than like voluntary action. At last many of the preferences for foods are induced from the interest for organoleptic characters or for the box it or the covering of the food.

The rigid and repetitive behaviors are one characteristic peculiar of these children: the continuous preference for the same food door to restrictions, monotony to feed, with consequent poor fundamental nourishing diet. An autistic child can insist in always eating the same food to every meal, can demand to expect a particular presentation of the food in the plate or only prefer foods with a reduced consistency a lot as purea or fruit juices can at last manifest preference refusal toward a particular food only for the color.(Nadom $G$ et al 2011)[2]

Great obstacle in the diet of these children is the inability to orally communicate their necessities, like the hunger, the satiety sense, the preference towards some foods for which it is necessary to carefully observe their reactions when to timetables, that they must rigorously be respects to you, comes assumed the food. The autistic child moreover has the tendency to isolate itself, has a reduced interaction with the contemporaries, rendering in such a way the much difficult learning of common food behaviors as the appropriate use of the spoon or the ability to nourish itself alone.The autistic syndrome behavior is often accompanied to numerous annoying symptoms of the gastrointestinal system, like abdominal pain, nausea, vomiting, constipation or diarrhoea, that they provoke to lack of appetite and malabsorption of the food. Such problems are often associated with one imbalance of intestinal microflora: in the faeces of autistic ones have been finds clostridi, enterococci, beyond quantitative differences of staphylococci and candid (Ekiel a. et al. 2010)[3]. In particular in the faecal flora of autistic patients has been found a greater presence of the Clostridium group histolyticum, clusters Clostridium I and II, that they are producers of a toxin in charge of the intestinal dysfunction, (Helena M.R. T.et al .2005)[4] with their metabolic products exercising also effects arranges to us. Monitoring and stabilization of intestinal microflora and knowledge on the role of particular strains in the aetiology of disturb autistic can increase to the probabilities of one appropriate therapy

\section{Nutritional biotherapy}

The nutritional principles of which the aliment is constituted are to all familiar ones: protein, fat, carbohydrates, mineral salts and vitamins. The protein they are essential for the growth and cellular renewal and have plastic function and of support for the organism; the carbohydrates they are the main suppliers of energy; the lipids constitute a reservoir energetic to consume in particular moments of necessity; the vitamins and the minerals are the catalysts of biochemical reactions that happen in our organism. These constituent foods assumed in correct quantities contribute to the good health and good functionality of our organism. But it is not sufficient to know the chemistry of foods in order to induce the individuals to one chosen rations them and satisfactory, because numerous other factors they influence the desire of a data food. Much importance, beyond to the aspect of a determined food, comes given to the preparation and to their presentation; the foods can be consumes to you cooked or raw and this possibility does not have to be underrated in how much the raw foods have the highest potentialities nutritional and energetic and therefore that partially they come instead lost with the conservation and the baking.

The baking is a methodical one of treatment of foods that can induce of the important modifications much for how much it not only concerns the outer aspect, but above all as far as the taste. In order to safeguard our health, the choice of food, the amount is indispensable to not only, but alsot o know to prepare them in adapted way for the aim to maintain their composition unchanged.

During the last few years the food concept has endured one radical transformation until the point to attribute to foods, beyond to their nutritional and sensory property, also an important role on the maintenance of the health, the psycho-physical well-being and the prevention of some pathologies. Sure various they are the factors that have contributed to this evolution on the new interpretation of the feeding and sure an important contribution is derived from the numerous scientific research that in the last decade they have evidenced, with abundance of data experiences them, the tight tie that exists between the feeding and the health.

The Nutritional Biotherapy has rediscovered the importance of the food and their combinations in the interaction that they have with organic metabolism. To second of the dysfunctions it is necessary to stimulate or protect an organ rather than another, but above all must keep in mind who the same foods, variously deals to you, they can have various therapeutic effects much (Arcari Morini D.et al,2005)[5]. With a wrong feeding infinites can be provoked disturb while maintaining an equilibrium alimentary the scope is caught up to nourish the organs and the woven ones in adequate way

The Nutritional Biotherapy proposes food like medicine, but it must hold account that exists one fundamental difference: while the medecine, for to the presence in it of or more active principles in high concentrations only can be assumed without risk for the limited period of the disease, the food can daily be 
assumed during the life, without some risk of harmful side effects. The Foods don't cure but they prevent the insorgence of chronic pathologies and consumes in the just proportions they maintain in the time the metabolic equilibrium to you, contribute to the good operation of organs and strengthen natural defense. Every food possesses in fact some characteristics that, beyond differentiating it from the others, determine, once consumed, an energetic modification, in the individual, different for every variable individual and from food to food.

The ingested foods not only influence on the physicist, but also on the behavior of the individual favoring the good humor, the aggressiveness, the concentration, concilianting the sleep, provoking excitation. It is known to all that the milk one conciles the sleep, containing substances with sedative action; the meat for its elevated energetic power renders aggressive, the fish thanks to its contained phosphorus elevated one, stimulates the brain and favors the learning; the salamis ones induce nervousness and insomnia; the grain paste generates calm and serenity. These little relative examples you to food of wide consumption indicate how much are important to know to choose the food adapted for the requirements of everyone for being able at best to carry out the own activities without damage for the health.

Sure various they are the factors that have contributed to this evolution on the new interpretation of the feeding and sure an important contribution is derived from the numerous scientific researches that in the past decade they have evidenced, with abundance of data experiences them the tight tie that exists between feeding and health. The appraisal of the salutistic potentiality and/or the prevention of one determined pathology sure represents the more delicate and critical phase in the valorization of food to the aim to consider it therapeutic. In the phase it begins them of the search, it must perfectly know the chemical composition of the food, and verify the existence of one whichever shape of interaction between or more components of the same food with one determined function of the human organism:not sure what easy and not always possible to demonstrate with scientific rigor. However, adequately, it arranges to you, the food can replace drugs and to cure many diseases, because contained natural molecules in the effective food are how much the medicines, but, what a lot important to consider, the food alimony is lacking also in the long term in effects collaterals. The scientific research must have as objective to find with exactitude the existing correlation between the assumption of a having food the ability to upgrade one determined function of the organism, (like to increase of tt the defenses in the comparisons of an excessive production of free radicals-oxidative stress), and the variation of one or more biomarkers that are linkable to the function to upgrade (Barazzoni $\mathrm{R}$ et al, 2014)[6].

For oxidative stress parameters like the anti-oxidant ability will be able to be used total of plasma (TAS); the determination of plasma concentrations of the sulphydryl groups, or the determination in the plasma of the active substances members of the food substances to the aim to estimate the bioavailability of the same food, namely its ability to transfer in the plasma and its active components so that can carry out therapeutic action. It is recognized for biophenols of the red wine and in particular to resveratrol, the preventive effect estimate comparisons of the cardiovascular diseases: as an example, the healthy potentiality could be evidenced or by means of epidemiological studies or demonstrating the inhibition of a biological process that is thought it can contribute to the insorgence of the pathology, like the inhibition of the oxidation of LDL.(Richer S..et al, 2013) [7]

Moreover, the influence of food matrix must be considered on the gastrointestinal absorption of the active substances and therefore of their bioavailability: many of they also turning out active in vitro, do not show some beneficial action when they are administered in vivo, because of one insufficient bioavailability. In recent more years, increasing evidences have been obtained on the priority role of the diet and of specific foods in the prevention and the therapy of many morbose conditions therapeutic. Foods they are daily assumed, but of recent it has only been possible to know the mechanism of action of their constituent ones.

Particular attention has been therefore turned to the chemical composition of the food and under consideration of particular activities demonstrated not only from single proteins, sugars and fatty acids, but also of compounds present in small concentrations, but particularly active like flavonoids, carotenoids, lycopene, polyphenols, indoles, and quercetin. The fruits and the vegetables are well known rich sources of anti-oxidant and nourishing. It is becoming more and more puts into effect them and important, just in order to take advantage of at best the property, the appraisal of the anti-oxidant power of the fruit and the vegetables but above all the appraisal of substances present anti-oxidants in fruits not commonly edible but from which molecules are obtained for extraction powerful.

The ORAC test (ORAC = Oxygen Radical Absorbance Capacity) is the specific method for the measurement of anti-oxidant power of the substances in biological samples and has been applied both is for fruits which blueberry, strawberry, raspberry, orange, red grape, cherry, grapes and for vegetables which spinach, Brussels sprouts, celery. It has been looked at then that green tea and black tea possess the highest value ORAC on peroxides regarding all the other vegetables.

The nutritionists which advice against to use in high doses some substances known them like fats, salts and sweets, but much more harmful they are considered food additives, not-nourishing substances added to foods in order to extend of the conservation, in order to facilitate the manufacture and in order to improve the 
taste or the aspect ( Cerutti,2006)[8]. The additives comprise the aromatizing and the flavour enhancers, the stabilisers, the thickeners, the emulsifiers, the colorings, the preservatives, the anti-oxidants, the sweeteners, the whiteners and the polishing agents, if every country has different laws for the regulation of the use of additives food, all convene on the emergency and the dosage for their employment. The tests which new additive comes every subordinate are not always foolproof; the results of the tests on animals they can also not correspond to those on the men, and are little famous the effects in the long term; some additives can provoke cancer, but it is difficult to go back the causes of a disease that employs years to develop itself; disowned it is moreover the interaction mechanism with other chemical substances of the food.

\section{Intestinal dysbiosis}

The intestinal dysbiosis is a pathological condition of the intestine characterized from an alteration of the native bacterial flora is one that lives in symbiosis with the man, collaborating to the digestion, regulating the permeability of the intestinal barrier, helping in the defense from the infections. One of the peculiar activities of these useful bacteria to the man is that one to prevent the proliferation of pathogenic bacteria whose increase damages the organism carrying to an imbalance in the immune system.

The lack of the bacteria benefits cause like necessary consequence one defective digestion and malabsorption of the food, with consequent formation of undigested residual food: the defective digestion of gluten and casein even generates opiate substances like gluteomorphine and caseomorphine. Just the pathogenic agents are cause to directly produce toxins that pass into the blood circle and arrive to the brain and the other organs, are to modify the intestinal barrier, creating of the true and own holes through which to the not digested toxins and the residual ones of the food more easy pass. This increased joined intestinal permeability to the defective digestion makes yes that food particles not perfectly digested child's step in the blood priming allergic and autoimmune reactions (D' Eufemia P. et al. 1996)[9]. The yeasts like Candida feed of the carbohydrates producing itself in the course of their digestion alcohol and acetaldehyde, two highly toxic substances for the organism.

The absence of bacteria benefits causes the lacked production of vitamins of the group B vitamins and vitamin $\mathrm{K}$, and in sure cases deficiency of iron and sulfur. The bad digestion and the malabsorption of the nourishing substances cause lack of zinc, magnesium, vitamin C. The deficiency of vitamins and of knows them minerals beyond to the presence of toxic substances of bacterial origin, is the main causes of difficulty of learning disabilities and concentration, irritability and nervousness.(Kidd PM .2000)[10].Bacterial flora in the intestine crasse metabolically introduces diversified and complex. Its primary function is to recover energy from carbohydrates not digested in theadvanced intestine, through the fermentation and the absorption of main products constituted from fatty acids to short chain, that they represent the $40-50 \%$ of the energy available of the carbohydrate.

The main fat acids to short-chain, acetate, propionate and butyrate, are metabolized in the colon, in the liver and in the intestinal mucosa; the single microorganisms of the colon like the bacteria, sulfate-reducers clostridia, bifidobacteria, answer selective to specific alimentary components; they have an important role in the synthesis of vitamins B and $\mathrm{K}$, and in the metabolism of bile acids, sterols and xenobiotics (Cummings $\mathrm{J} \mathrm{H}$, et al. .1997)[11]

Many studies are made in order to explain the possibility of one logon intestine-brain in autistic children (Horvath K.,2002; KuddoT, 2003; Reichelt KL .2009)[ 12-14] in order to explore the biological basis of this disturbance, but the aetiology remains unknown. Recent publications they have focused attention on the gastrointestinal function and the morphology of these children; they have been marked histological anomalies of the esophagus, the stomach ,the tenuous intestine and the colon, dysfunction of the hepatic functionality and intestinal permeability.

It is in the children who in the adults are obvious a continuous inflammatory state of the intestinal tract with an increase of the protein presence in urines, connected to behavioural manifestations which difficulty of concentration and attention, hyperactivity, attacks of panic anxiety and other problems. While a balanced diet favors the proliferation of the bacteria benefits that help to digest and to produce to vitamins and amino acids, an inadequate feeding door to an increase of pathogenic bacteria. Currently it is thought that the alterations of intestinal flora are cause of many chronic and degenerate diseases.

Not only syndrome of the irritable intestine, disturbs inflammatory of the intestine, arthritis rheumatoid and spondilosite anchylosing is pathological conditions that have been connected to alterations of the intestinal microflora but also nasopharyngeal infections, allergic diseases, asthma, autism and schizophrenia. The children with manifestations of atopic eczema suffer from disturb psychological in double measure of children without this pathology: of it follows a sensitive correlation between the two conditions is uncovered that factors which antibiotics, physical and psychical stress, pollution, heavy metals and sure components of the diet contribute to intestinal dysbiosis. If these causes can be eliminated or at least reduced, then the aimed cures to modify the microflora can be more effective. 


\section{Conclusions}

The treatment of the digestive problems can have positive effects you on behavior of the autistic subjects, but is necessary to teach those who assists these persons as to choose the food and like making to accept them. The first step begins with the application of some behavioural techniques (Kodak T, Piazza CC, 2008)[15,]: in fact it has been evidenced from a descriptive analysis of selective subjects in the comparisons of the food, than the more frequent reactions of the children are those to abandon the table or not to show themselves interested. Valdimarsdottir $\mathrm{H}$. and coll

(2010)[16] they tried with behaviors alternated, which not to allow to you to go away from the table or to leave the fork, noticing that in the autistic children the opposition to the food diminished and began to taste. Not only the largeness of the mouthful, the type of food, the present amount in the plate, the consistency, the color, the sapore and the shape of the food, but also the type of used tool, the timetable established for the meal, the atmosphere, the presence of relatives, can influence the alimentary behavior of the autistic ones.

Mueller MM and coll (2004)[17] demonstrated, experimenting on two autistic children, then, stirring the preferred food with that one not preferred and gradually increasing the proportion of the not preferred one in the prescription, increased the consumed food variety. This discovery agrees with studies that demonstrate like the acquaintance of a new taste stirred to that one already known and preferred door towards one preference also of the new taste. Other methods exist also in order to condition the preference and the aversion of the tastes as the manipulation of the food varying the aspect and the consistency so as to expand the variety of the acceptable food for the autistic child.

Besides, the autistic syndrome, often there and the grip logon with the alimentary intolerances. The autistic children are intollerant to the protein of the milk ones of cow and to the gluten, because of a genetic digestive enzyme deficit specific; therefore if a food completely is not digested in all its principles, then it can cause an adverse reaction of the immune system of the intestine. Such reaction verification mainly if an internal inflammation is present and can be involved one instantaneous allergic reaction (mediated IgE antibodies) or delayed also 1-2 days (mediated not IgE). The typical symptoms of the allergy to the food are limit you to the gastrointestinal feature mainly, but they can be involved headaches, respiratory problems and in serious cases, anaphylaxis.

More and more frequently it is watched to the exposure to the toxic metals like cause of dysfunction of the learning and the behavior in the children. Several searches have correlated the exposure to the metals, like the lead and mercury, condition in a position to altering the cerebral development in the young age. The investigators have found lacked deficiencies soccer, branch, zinc and chromium in the autistic children, beyond, the mineral deficiencies render the more susceptible child to the absorption of the toxic metals. Deficiencies of magnesium are associated to disturb of the attention and iperactivity: the association magnesium/B6 vitamin is from considering one of the more effective treatments for the improvement of the symptom. Because, enzimatic deficiencies many children introduce difficulty in the digestion, carbohydrate absorption. and proteins: these anomalies closely are tied to the unexpected changes of nocturnal behavior, irritability, aggressiveness and awakenings.The amino acids are necessary in order to produce neurotransmitters and are in a position to influencing are the humor that the behavior and some are mainly implied to you in the autistic syndrome: thryptophan, glutamic acid, aspartic acid, phenylalanine, carnosine, 1-methylhistidine, $\beta$-alanine, methionine, taurine, and cysteine. Also, badly an absorption of lipids, pancreatic dysfunctions, and elevated bacterial increase, are given easy found in the autism. More and more it is aware that essential the fat acids, are indispensable for the development of the brain, therefore they are particularly important for the suffering children for disturbs of the development like the autism. A study carried out on autistic children evidenced a $20 \%$ in less of the level than fat acid $\omega_{3} \square$ respect to a control group. In particular the level of decosaesanoico acid (DHA) turned out inferior of $23 \%$. These deficiencies provoked to a meaningful increase of the relationship $\omega_{6} / \omega_{3}$, relationship that normally must oscillate between 1 and 4 . Also the amount of ingested liquids must be controlled and eventually integrated with many vitamins (Seiverling LJ,2011)[18].

Beyond to the paediatrist and psycotherapist therefore the parents they would have to be always taken advantage of the support of a nourishist/dietologist: the diet must be adapted is in calories that macro and micronourishing and must be calculated based on the necessities characterize them of the child, holding present its alimentary preferences, the atmosphere in which it is found and eventually its activities

\section{References}

[1]. Delacato C.H. Alla scoperta del bambino autistico, Armando editore,Roma,1996

[2]. Nadon G, Feldman DE, Dunn W, Gisel E Problemi dei pasti in bambini con disturbo dello spettro autistico e dei loro fratelli in genere in via di sviluppo: uno studio di confronto. .Autismo 15 (1) 2011, 98-113

[3]. Ekiel A. Aptekorz M, Kazek B, Wiechuła B, Wilk I, Martirosian G. Intestinal microflora of autistic children Med Dosw Mikrobiol. 62(3) 2010, 237-43. 
[4]. Helena M. R. T. Parracho, Max O. Bingham, $\dagger$ Glenn R. Gibson and Anne L. McCartney Differences between the gut microflora of children with autistic spectrum disorders and that of healthy children Journal of Medical Microbiology 54, 2005, 987-991

[5]. Arcari Morini D, D’Eugenio A.,Aufiero F.Il potere farmacologico degli alimenti RED edizioni, Milano,2005

[6]. Barazzoni R, Silva V.,Singer P. Clinical biomarkers in metabolic sindrome Nutr. Clin Prat 29 (2),2014, 215-221

[7]. Richer S.,Stiles W.,Ulanski L., Caroll D.,Podella C.Observation of human retinal remodelling in octogenarians with a resveratrol based nutritional supplement. Nutrients 5(6), 2013, 1989-2005

[8]. Cerutti G. Residui,additivi e contaminanti degli alimenti Tecniche nuove( II ed), 2006

[9]. D’Eufemia P., Celli M, Finocchiaro R.,Pacifico L.,Vizzi L.,Zaccagbini M.,Cardi E.,Giardini O.,Abnormal intestinal permenility in children with autism Acta Paediatr 85(9),1996, 1076-9

[10]. Kidd PM Attention deficit/hyperactivity disorder (ADHD)in children: rationale for its integrative management Altern.Med. Rev. 5(5), 2000, 402-28

[11]. Cummings JH,Macfarlane GT. Role of intestinal bacteria in nutrient metabolism J.Pentearral Nutr.21 (6),1997, 357-65

[12]. Horvath K,Parman JA Autistic disorder and gastrointestinal disease Curr Opin Pediatr 14 (5),, 2002, 583-7

[13]. Kuddo T,Nelson KB How common are gastrointestinal disorders in children with auyism? Curr opin Pediatr 15(3), 2003, 339-43

[14]. Reichelt KL,Knivsberg AM,The possibility and probability of a gut -to-brain connection in autism Ann.Clin. Psychiatry 21(4),2009 205-11

[15]. Kodak T, Piazza C.C. Assessment and behavioral treatment of feeding and sleeping disorders in children with autism spectrum disorders. Child and Adolescent Psychiatric Clinics of North America. 17, 2008,887-905

[16]. Valdimarsdottir H, Halldorsdottir L.Y, Sigurdardottir Z.G. Increasing the variety of foods consumed by a picky eater: Generalization of effects across caregivers and settings. Journal of Applied Behavior Analysis. 43, 2010,101-105

[17]. Mueller M.M, Piazza C.C, Patel M.R, Kelley M.E, Pruett A. Increasing variety of foods consumed by blending nonpreferred foods into preferred foods. Journal of Applied Behavior Analysis. 37, 2004, 159-170

[18]. Seiverling LJ,Williams KE,Ward-Horner J,Sturemey P. Interventions to treat feeding problems in children with autism spectrumm disorder: a comprehensive rewiew. Autism and child Psychopathology Series 2011, 491-508 